\title{
Motivational Needs-Driven Mobile Phone Design
}

\author{
Judy van Biljon ${ }^{1}$, Paula Kotzé ${ }^{1}$, and Gary Marsden ${ }^{2}$ \\ ${ }^{1}$ School of Computing, University of South Africa, South Africa \\ ${ }^{2}$ Department of Computer Science, University of Cape Town, South Africa \\ \{vbiljja, kotzep\}@unisa.ac.za, gaz@cs.uct.ac.za
}

\begin{abstract}
This paper provides support for the use of motivational needs in identifying mobile phone uses and related features. Drawing on motivational human and usage space research, the findings of interviews and surveys, this paper proposes the Mobile phone Usage Space Model (MUSM). MUSM distinguishes between two groups of features by identifying necessary and additional features, thus focusing the designer's activity on motivational needsdriven design, rather than feature escalation that currently appears to dominate.
\end{abstract}

Keywords: Mobile phone design, usage spaces, motivational needs.

\section{Introduction}

This paper addresses the issue of user needs in the context of mobile phone design. In Human-Computer Interaction (HCI) user needs are usually studied from task and context-oriented perspectives. This approach works well in designing systems where specified and structured tasks are involved. Since mobile phones are personal devices not strictly fitting the task domain, we argue that a different approach is required in designing such devices and the use options available. The feature driven approach has been widely used to design mobile phones this far [1]. Feature addition is driven by the need to increase the demand and desirability of the product, but in reality it may reduce usability, and tends to be counter-productive as many users find it difficult to cope with the information overload and the cognitive demands of mobile phone technology [2, 3]. If adding features does not satisfy user needs, what does? Focusing on motivational needs can provide designers with a new alternative to the current feature-driven approach. Section 2 sets theoretical foundations by highlighting feature-driven research, motivational human needs and the representation of mobile phone uses. Section 3 describes our research to investigate links between user needs and mobile phone features. Section 4 concludes.

\section{Theoretical Framework}

Much research on mobile phones in the HCI arena is directed at finding the set of key features around which the user interface should be optimised for each target group. Han et al. [1], for example introduced an approach to identifying both desirable and undesirable properties of critical design features and developed empirical models to 
link design features to satisfaction levels. Ling and Hwang [4] considered specific features and found that only colour display and Internet browsing features improves overall satisfaction significantly. All these studies have an inherent limitation: they start with specific design elements, features or functions and then try to establish the value these features have for users. The number of features increase and it is impractical to study all the different combinations of features to produce reliable results.

An alternative perspective is to consider theories on human needs. One of the first theories on motivational needs was proposed in 1954 by Maslow, which he later expanded to a total of eight levels of human needs [5]. The needs are: physiological; safety and security; belongingness; esteem; cognitive; aesthetic; self-actualization; and self-transcendence. The Institute for Management Excellence [6] proposes an alternative set of needs namely: security; adventure; freedom; exchange; power; expansion; acceptance; community; and expression. Herzberg's motivator-hygiene theory from the field of industrial psychology [7], discriminates between factors which are referred to as motivational versus those called hygiene. Hygiene factors meet physiological, safety and social needs in the workplace, while motivational factors encourage job satisfaction and appeal to human needs of growth and selfadvancement [8]. Applied to mobile phone features, motivational factors would serve as a discriminator for the prospective buyer. Hygiene factors will have to be present to satisfy basic requirements while features in the motivational group can be used to customise the device for specific users. To be useful to designers, user needs have to be linked to features via uses or usage spaces. The usage space model by Marcus and Chen [9] provides a starting point for presenting mobile device uses.

\section{Research Methodology and Findings}

We investigated the link between motivational needs and mobile phone features using a questionnaire driven study. The questionnaire was based on a literature study and was verified in structured interviews (with 10 participants, gender balanced, age groups 20-29, 30-39, 40-49 and 50-59) from three ethnic groups (African, Asian and European). The following observations were made during the design and verification stage: participants over the age of 30 used less than $40 \%$ of the available features, thought of a mobile phone as a mobile version of the traditional phone, and identified relationship building and security as the highest use priorities. Participants under the age of 30 used between $40 \%$ and $50 \%$ of the features on their mobile phones, viewed the phone as a tool for communication, organization and entertainment but were inhibited by cost. The questionnaire was refined and tested in a pilot survey involving 39 participants, all questionnaires used are available [10].

Since past research (confirmed by our observations) indicated that age and technological development influences mobile phone use [11], we limited the age bracket and technological development level for our pilot survey and final survey to university students in computer science between the ages of 18 and 27. Using a refined version of the pilot survey questionnaire, the final survey involved 138 participants from two South African universities, $68 \%$ male and 32\% female. 
Two data reduction (statistical) methods were used to analyse the data collected during the final survey, namely exploratory factor analysis and optimal scaling. The aim of this analysis was to find whether features cluster together in terms of usage frequency. Exploratory factor analysis produced the following main usage clusters:

- New technology: Bluetooth, video player, MP3, e-mail, voice recorder, etc.

- Personal history: camera, photo-album, MMS, ring tones, personal notes, etc.

- Safety, security, relationships: phone book, caller identity, missed calls, SMS.

- Organisation: vibrating alert, reminders, profiles, calendar

Optimal scaling was applied to the same data to verify the clustering. On inspection of the graphs (not included here) feature clusters were selected based on proximity. The following main groupings (usage clusters) were identified:

- Safety, security, relationships: phone book, caller identity, missed calls; SMS.

- Organisation: vibrating alert, reminders, profiles and alarm.

- Personal history: camera, MP3, personal organiser, photo-album, video capture.

This means that two data reduction methods, i.e. exploratory factor analyses and optimal scaling, produced similar groupings, namely: safety and security, relationships, organisation, personal history. Other additional usage spaces emerged from the literature study and the interviews, namely: personal information, nonpersonal information, m-commerce, entertainment, image and expansion. Integrating the findings from our survey with those from the interviews and literature survey, we propose the MUSM (Mobile phone Usage Spaces Model) consisting of a set of core spaces and additional spaces applicable to this user group as depicted in Fig 1:

- Core (hygiene): relationships, personal information, organisation, safety, security.

- Additional (motivational): entertainment, m-commerce, expansion, non-personal information, personal history, image.

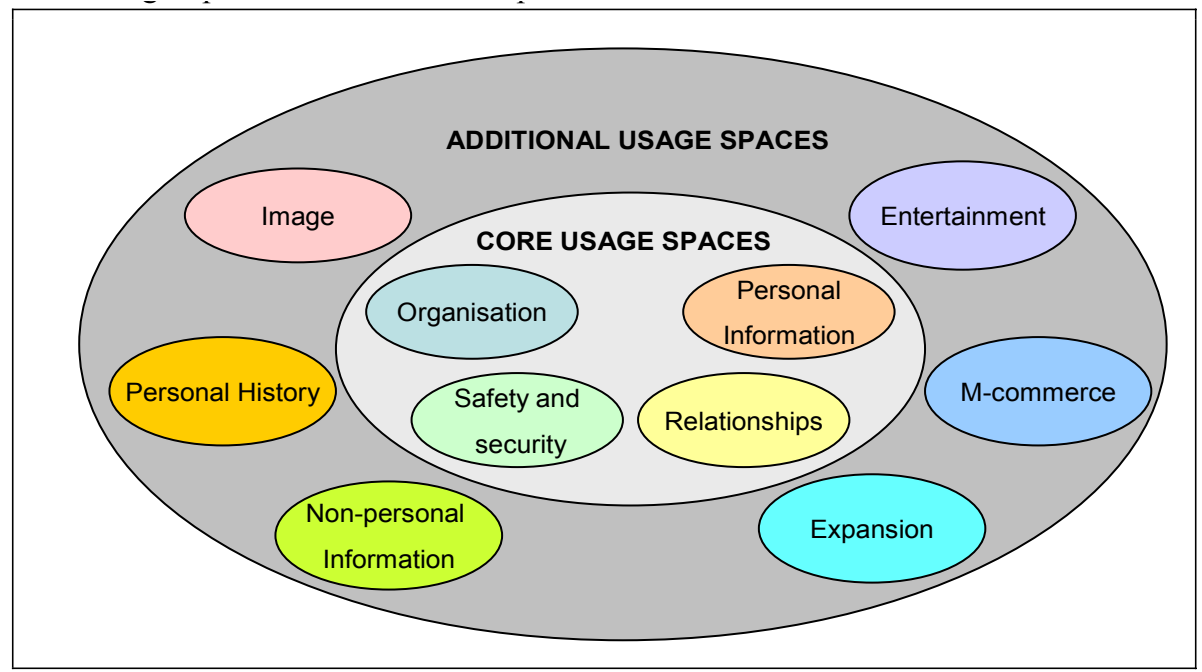

Fig. 1. Proposed usage space model with two categories of usage spaces 
The usage spaces identified correspond to four of the motivational human needs identified by Maslow [5] i.e. safety and security, belongingness (relationships), cognitive (personal and non-personal information, organisation) and self-actualisation (expansion, personal-history). Dividing usage spaces into core spaces and additional spaces is supported by Herzberg's motivator-hygiene theory [8]. Furthermore, this study provides empirical support for Chen and Marcus's model [9] (that was lacking until now) and extends their model by linking usage spaces to mobile phone features.

\section{Conclusion}

The main contribution of our research is linking the models of Maslow [5] and Herzberg [8] to that of Marcus[9], thereby grounding mobile phone feature selection in motivational needs as opposed to a market-driven, more-is-better approach which leads to redundant features and cognitive overload. MUSM allows users to express their mobile phone usage needs in non-technical terms, while designers can use the model as a guideline to convert the expressed user needs into a set of features that fit the motivational needs of the user. Further research is needed to refine MUSM. The features associated with each usage space will have to be adapted according to new developments and the grouping of core and additional spaces could change for different target (age) groups but the structure and usage spaces identified are grounded solidly in motivational needs theories.

\section{References}

1. Han, S.H., Kim, J.K., Yun, M.H., Hong, S.W., Kim, J.H.: Identifying Mobile Phone Design Features Critical to user Satisfaction. Human Factors and Ergonomics in Manufacturing 14(1), 15-29 (2004)

2. Palen, L., Salzman, M., Youngs, E.: Going wireless: behavior \& practice of new mobile phone users. In: Proceedings from the 2000 ACM conference on Computer supported cooperative work, ACM Press, Philadelphia, Pennsylvania (2000)

3. Ziefle, M., Bay, S.: The Complexity of Navigation Keys in Cellular Phones and their Effects on Performance of Teenagers and Older Adult Phone Users. In: HCI International 11th international conference, Las Vegas, Nevada, USA (2005)

4. Ling, C., Hwang, W.: Users Satisfaction with Five New Cell Phone Features. HCI International - 11th international conference, Las Vegas, Nevada, USA (2005)

5. Maslow, A., Lowery, R.: Towards a Psychology of Being, 3rd edn. Wiley, New York (1998)

6. Institute for Management Excellence (accessed June 2006), http://www.itstime.com/ jun97.htm

7. Löfgren, M., Witell, L.: Kano's Theory of Attractive Quality and Packaging. Quality management journal 12(3), 7-20 (2005)

8. Herzberg, F.: One more time: How do you motivate employees? Harvard Business Review 46(1), 53-62 (1968)

9. Marcus, A., Chen, E.: Designing the PDA of the future. Interactions 9(1), 34-44 (2002)

10. Van Biljon, J.A.: A model for representing the motivational and cultural factors that influence mobile phone usage, School of Computing.University of South Africa (2006)

11. Teo, T.S.H., Pok, S.H.: Adoption of the internet and WAP-enabled phones in Singapore. Behaviour \& Information Technology 22(4), 281-289 (2003) 